\title{
A SUB-FUNCTOR FOR EXT AND COHEN-MACAULAY ASSOCIATED GRADED MODULES WITH BOUNDED MULTIPLICITY
}

\author{
TONY J. PUTHENPURAKAL
}

\begin{abstract}
Let $(A, \mathfrak{m})$ be a Henselian Cohen-Macaulay local ring and let $\mathrm{CM}(A)$ be the category of maximal Cohen-Macaulay $A$-modules. We construct $T: \operatorname{CM}(A) \times \operatorname{CM}(A) \rightarrow \bmod (A)$, a subfunctor of $\operatorname{Ext}_{A}^{1}(-,-)$ and use it to study properties of associated graded modules over $G(A)=\bigoplus_{n \geq 0} \mathfrak{m}^{n} / \mathfrak{m}^{n+1}$, the associated graded ring of $A$. As an application we give several examples of complete Cohen-Macaulay local rings $A$ with $G(A)$ Cohen-Macaulay and having distinct indecomposable maximal Cohen-Macaulay modules $M_{n}$ with $G\left(M_{n}\right)$ Cohen-Macaulay and the set $\left\{e\left(M_{n}\right)\right\}$ bounded (here $e(M)$ denotes multiplicity of $M)$.
\end{abstract}

\section{INTRODUCTION}

Let $(A, \mathfrak{m})$ be a Henselian Noetherian local ring. Recall that $A$ satisfies KrullSchmidt property, i.e., every finitely generated $A$-module is uniquely a direct sum of indecomposable $A$-modules. Now assume that $A$ is Cohen-Macaulay. Then we say $A$ is of finite (Cohen-Macaulay) representation type if $A$ has only finitely many indecomposable maximal Cohen-Macaulay (MCM) $A$-modules. Auslander proved that in this case $A$ is an isolated singularity, for instance see [17, Theorem 4.22]. If in addition $A$ is equicharacteristic (containing a) perfect residue field then Dieterich and Yoshino (independently) proved that if $A$ is an isolated singularity and not of finite representation type then $A$ satisfies the first Brauer-Thrall conjecture (made for Artin algebra's), i.e., there exists indecomposable MCM $A$-modules $M_{n}$ with $\left\{e\left(M_{n}\right)\right\}_{n \geq 1}$ unbounded (here $e(M)$ denotes multiplicity of $M$ ), see 17, Theorem 6.2]. If $A$ is not an isolated singularity then it follows from work of Huneke and Leuschke [6, Theorem 1] that $A$ has indecomposable MCM $A$-modules $M_{n}$ such that $\left\{e\left(M_{n}\right)\right\}_{n \geq 1}$ is bounded. We call this property as weak Brauer-Thrall II.

Let $G(A)=\bigoplus_{n>0} \mathfrak{m}^{n} / \mathfrak{m}^{n+1}$ be the associated graded ring of $A$ and if $M$ is a finitely generated $A$-module then let $G(M)=\bigoplus_{n \geq 0} \mathfrak{m}^{n} M / \mathfrak{m}^{n+1} M$ be the associated graded module of $M$. Note that we will only take associated graded modules with respect to $\mathfrak{m}$. Assume $G(A)$ is Cohen-Macaulay. There are two natural questions that arise.

(1) Does there exist a non-free MCM $A$-module $M$ with Cohen-Macaulay associated graded module.

Date: August 22, 2018.

1991 Mathematics Subject Classification. Primary 13A30, 13C14; Secondary 13D40, 13D07.

Key words and phrases. Associated graded rings and modules, Brauer-Thrall conjectures, strict complete intersections, Henselian rings, Ulrich modules. 
(2) How many indecomposable MCM $A$-modules exist with Cohen-Macaulay associated graded modules. This naturally splits into two sub-questions:

(a) (Brauer-Thrall-I). Does there exist indecomposable MCM modules $\left\{M_{n}\right\}_{n \geq 1}$ with $G\left(M_{n}\right)$ Cohen-Macaulay and $e\left(M_{n}\right) \rightarrow+\infty$.

(b) (weak Brauer-Thrall-II) Does there exist distinct indecomposable MCM modules $\left\{M_{n}\right\}_{n \geq 1}$ with $G\left(M_{n}\right)$ Cohen-Macaulay and $e\left(M_{n}\right)$ bounded.

We now discuss what is previously known regarding these questions.

(1) This is known for $\operatorname{dim} A \leq 1$. In one of the preliminary results in this paper we settle the $\operatorname{dim} A=2$ case affirmatively. It is also easy to see that if $A$ has minimal multiplicity then every MCM $A$-module has Cohen-Macaulay associated graded module (for instance see [9, Theorem 16]). If $A$ is a strict complete intersection (i.e., $G(A)$ is also a complete intersection) and $A$ is a quotient of a regular local ring then $A$ has an Ulrich module $U$, see [5, 2.5]. Recall an Ulrich module $U$ is an MCM $A$-module if its multiplicity equals its number of minimal generators. It is well known that if $U$ is Ulrich then $G(U)$ is Cohen-Macaulay. If $A=\widehat{R}$ where $R$ is a two dimensional Cohen-Macaulay standard graded algebra (and a domain) over an infinite field then also $A$ has an Ulrich module, see [1, 4.8].

2(a) If $A$ is a Cohen-Macaulay isolated singularity, convergent power series ring over a perfect field and having minimal multiplicity and not of finite representation type then Brauer-Thrall-I holds for associated graded modules. In a previous work, the author proved that if $A$ is a complete equi-characteristic hypersurface ring (and an isolated singularity) with algebraically closed residue field and even dimension (and of infinite representation type) then there exists indecomposable Ulrich $A$ modules $\left\{M_{n}\right\}$ with $\left\{e\left(M_{n}\right)\right\}_{n \geq 1}$ unbounded, see [14, 1.11].

2(b) An easy case when this holds is when $A$ has minimal multiplicty and is not an isolated singularity. To the best of the authors knowledge there is no other previous work discussing weak Brauer-Thrall II for associated graded modules. The main goal of this paper is to give examples of Cohen-Macaulay local rings satisfying weak Brauer-Thrall II i.e., for the existence of distinct MCM $A$-modules $M_{n}$ such that $G\left(M_{n}\right)$ is Cohen-Macaulay and $\left\{e\left(M_{n}\right)\right\}_{n \geq 1}$ is a bounded set.

We show that the following classes of Henselain Cohen-Macaulay local rings $A$ with $G(A)$ Cohen-Macaulay satisfy weak Brauer-Thrall II

(i) $\operatorname{dim} A=1,2$ and $A$ is not an isolated singularity; see Theorem 9.7

(ii) Let $(Q, \mathfrak{n})$ be a Henselian regular local ring and let $A=Q /\left(f_{1}, \ldots, f_{c}\right)$ be a strict complete intersection. Let $f_{1}=g^{i} h$ with $g$ irreducible, $(h$ is possibly a unit if $i \geq 2$ and is a non-unit if $i=1$ ) and $g$ does not divide $h$. If $i \geq 2$ assume $\operatorname{dim} A \geq 1$. If $i=1$ assume $\operatorname{dim} A \geq 2$; see Theorem 8.1

(iii) Let $(R, \mathfrak{n})$ be a Cohen-Macaulay local ring having a non-free MCM module $E$ with $G(E)$ Cohen-Macaulay. Also assume $G(R)$ is Cohen-Macaulay. Let $r \geq 1$ and let $B=A\left[\left[X_{1}, \ldots, X_{r}\right]\right]$ or $B=R\left[X_{1}, \ldots, X_{r}\right]_{\left(\mathfrak{n}, X_{1}, \ldots, X_{r}\right)}$. Note $G(B)$ is CohenMacaulay. Let $0 \leq l \leq r-1$ and let $g_{1}, \ldots, g_{l}$ be such that $g_{1}^{*}, \ldots, g_{l}^{*}$ is $G(B)$ regular. Set $A=B /\left(g_{1}, \ldots, g_{l}\right)$; see Theorem 10.3 .

Construction used to prove our results:

Let $(A, \mathfrak{m})$ be a Cohen-Macaulay local ring and let $\operatorname{CM}(A)$ be the category of maximal Cohen-Macaulay $A$-modules. We construct $T: \operatorname{CM}(A) \times \mathrm{CM}(A) \rightarrow \bmod (A)$, a sub-functor of $\operatorname{Ext}_{A}^{1}(-,-)$ as follows: 
Let $M$ be a MCM $A$-module. Set

$$
e_{A}^{T}(M)=\lim _{n \rightarrow \infty} \frac{(d-1) !}{n^{d-1}} \ell\left(\operatorname{Tor}_{1}^{A}\left(M, \frac{A}{\mathfrak{m}^{n+1}}\right)\right) .
$$

This function arose in the authors study of certain aspects of the theory of Hilbert functions 9, 10]. Using [9, Theorem 18] we get that $e_{A}^{T}(M)$ is a finite number and it is zero if and only if $M$ is free. Let $s: 0 \rightarrow N \rightarrow E \rightarrow M \rightarrow 0$ be an exact sequence of MCM $A$-modules. Then by [12, 2.6] we get that $e_{A}^{T}(E) \leq e_{A}^{T}(M)+e_{A}^{T}(N)$. Set $e^{T}(s)=e_{A}^{T}(M)+e_{A}^{T}(N)-e_{A}^{T}(E)$.

Definition 1.1. We say $s$ is $T$-split if $e_{A}^{T}(s)=0$.

Remark 1.2. Here $T$ stands for Tor.

Definition 1.3. Let $M, N$ be MCM $A$-modules. Set

$$
T_{A}(M, N)=\{s \mid s \text { is a } T \text {-split extension }\} .
$$

We show

Theorem 1.4. (with notation as above) $T_{A}: \operatorname{CM}(A) \times \operatorname{CM}(A) \rightarrow \bmod (A)$ is a sub-functor of $\operatorname{Ext}_{A}^{1}(-,-)$.

It is not clear from the definition whether $T_{A}(M, N)$ is non-zero. Our next results shows that there are plenty of $T$-split extensions if $\operatorname{dim}_{\operatorname{Ext}_{A}^{1}}(M, N)>0$. We prove

Theorem 1.5. Let $(A, \mathfrak{m})$ be a Cohen-Macaulay local ring and let $M, N$ be $M C M$ A-modules. Then

$$
\operatorname{Ext}_{A}^{1}(M, N) / T_{A}(M, N) \text { has finite length. }
$$

1.6. If $s: 0 \rightarrow N \rightarrow E \rightarrow M \rightarrow 0$ is an extension of MCM $A$-modules then we have a complex of $G(A)$-modules

$$
G(s): 0 \rightarrow G(N) \rightarrow G(E) \rightarrow G(M) \rightarrow 0 .
$$

The utility of $T$-split sequences is

Lemma 1.7. (with hypotheses as in 1.6) Assume $s$ is $T$-split and $G(N)$ is CohenMacaulay. Then $G(s)$ is exact. In particular if $G(M)$ is also Cohen-Macaulay then $G(E)$ is Cohen-Macaulay.

Lemma 1.7 is used to prove the following main technical result in our paper.

Theorem 1.8. Let $(A, \mathfrak{m})$ be a Henselian Cohen-Macaulay local ring of dimension $d \geq 1$. Suppose $M, N$ are $M C M$ modules with $G(M), G(N)$ Cohen-Macaulay. If there exists only finitely many non-isomorphic MCM A-modules D with $G(D)$ Cohen-Macaulay and $e(D)=e(M)+e(N)$; then $T_{A}(M, N)$ has finite length (in particular $\operatorname{Ext}_{A}^{1}(M, N)$ has finite length). If $h$ is the number of such isomorphism classes then $\mathfrak{m}^{h}$ annihilates $T_{A}(M, N)$.

Remark 1.9. Notice statement of Theorem 7.1 is formally similar to the statement of a result by Huneke and Leuschke [6, Theorem 1]. The proof is similar too, except in few details which we describe in proof of this result in section 7 .

All our results follow by constructing suitable MCM modules $M, N$ with $G(M)$,

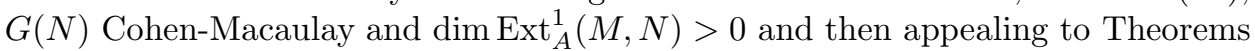
1.5 and 1.8. Our techniques also enable us to discuss weak Brauer-Thrall for Ulrich modules; see section 10 . 
Remark 1.10. Let $I$ be an $\mathfrak{m}$-primary ideal and let $G_{I}(A)=\bigoplus_{n>0} I^{n} / I^{n+1}$ be the associated graded ring of $A$ with respect to $I$. Now suppose $G_{I}(A)$ is CohenMacaulay. Then we can ask questions similar to the case when $I=\mathfrak{m}$. However our technique fails in this case. See remark 3.2 for an explanation.

We now describe in brief the contents of this paper. In section two we discuss some preliminary results that we need. In section three we prove Theorem 1.4 In the next section we prove Theorem 1.5. In section five we discuss a construction made in [11. In the next section we prove Lemma 1.7. In section seven we prove Theorem 1.8 and two results analogous to it. In the next three section we give our examples showing existence of weak Brauer-Thrall-II for a large class of rings.

\section{Preliminaries}

In this section we discuss a few preliminaries that we need. Throughout all rings are commutative Noetherian and all modules considered are finitely generated unless otherwise stated. The length of an $A$-module $M$ is denoted by $\ell(M)$ while $\mu(M)$ denotes the number of its minimal generators.

2.1. Let $(A, \mathfrak{m})$ be a local ring. Let $N$ be a $A$-module of dimension $r$. It is well-known that there exists a polynomial $P_{N}(z) \in \mathbb{Q}[z]$ of degree $r$ such that $P_{N}(n)=\ell\left(N / \mathfrak{m}^{n+1} N\right)$ for all $n \gg 0$. We write

$$
P_{N}(z)=\sum_{i=0}^{r}(-1)^{i} e_{i}(N)\left(\begin{array}{c}
z+r-i \\
r-i
\end{array}\right) .
$$

Then $e_{0}(N), \cdots, e_{r}(N)$ are integers and are called the Hilbert coefficients of $N$. The number $e_{0}(N)=e(N)$ is called the multiplicity of $N$. It is positive if $N$ is non-zero. The number $e_{1}(N)$ is non-negative if $N$ is Cohen-Macaulay; see [9, Proposition 12]. Also note that

$$
\sum_{n \geq 0} \ell\left(N / \mathfrak{m}^{n+1} N\right) z^{n}=\frac{h_{N}(z)}{(1-z)^{r+1}}
$$

where $h_{N}(z) \in \mathbb{Z}[z]$ with $e_{i}(N)=h_{N}^{(i)}(1) / i$ ! for $i=0, \ldots, r$.

2.2. Let us recall the definition of superficial elements. Let $N$ be an $A$-module. An element $x \in \mathfrak{m} \backslash \mathfrak{m}^{2}$ is said to be $N$-superficial if there exists $c>0$ such that $\left(\mathfrak{m}^{n+1} N: x\right) \cap \mathfrak{m}^{c} N=\mathfrak{m}^{n} N$ for all $n \gg 0$. It is well-known that superficial elements exist when the residue field $k$ of $A$ is infinite. If depth $N>0$ then one can prove that a $N$-superficial element $x$ is $N$-regular. Furthermore $\left(\mathfrak{m}^{n+1} N: x\right)=\mathfrak{m}^{n} N$ for all $n \gg 0$.

Let $\operatorname{dim} N=r$. Then a sequence $x_{1}, \ldots, x_{s} \in \mathfrak{m}$ (where $s \leq r$ ) is called an $N$-superficial sequence if $x_{i}$ is $N /\left(x_{1}, \ldots, x_{i-1}\right) N$-superficial for all $i$.

2.3. Minimal reduction: For this notion we assume that the residue field of $A$ is infinite. Let $\operatorname{dim} N=r \geq 1$. We say $J=\left(x_{1}, \ldots, x_{r}\right)$ is a minimal reduction of $N$ if $\mathfrak{m}^{n+1} N=J \mathfrak{m}^{n} N$ for all $n \gg 0$.

Assume further that $N$ is Cohen-Macaulay. Then it can be easily shown that if $x_{1}, \ldots, x_{r}$ is an $N$-superficial sequence then $J=\left(x_{1}, \ldots, x_{r}\right)$ is a minimal reduction of $N$. 
2.4. Behavior of Hilbert coefficients with respect to superficial elements: Assume $N$ is an $A$-module with depth $N>0$ and dimension $r \geq 1$. Let $x$ be $N$-superficial. Then by [9, Corollary 10] we have

$$
e_{i}(N / x N)=e_{i}(N) \text { for } i=0, \ldots, r-1 .
$$

2.5. Sally Descent: Assume depth $M \geq 2$ and $x$ is $M$-superficial. Set $N=M / x M$. If depth $G(N) \geq 1$ then $\operatorname{depth} G(M) \geq 2$; see [9, Theorem 8(2)].

2.6. Let $M$ be an $A$-module. We denote it's first syzygy-module by $\Omega(M)$. If we have to specify the ring then we write it as $\Omega_{A}(M)$. Recall $\Omega(M)$ is constructed as follows: Let $G \stackrel{\phi}{\rightarrow} F \stackrel{\epsilon}{\rightarrow} M \rightarrow 0$ be a minimal presentation of $M$. Then $\Omega(M)=$ $\operatorname{ker} \epsilon$. It is easily shown that if $G^{\prime} \stackrel{\phi^{\prime}}{\rightarrow} F \stackrel{\epsilon^{\prime}}{\rightarrow} M \rightarrow 0$ is another minimal presentation of $M$ then $\operatorname{ker} \epsilon \cong \operatorname{ker} \epsilon^{\prime}$.

Set $\Omega^{1}(M)=\Omega(M)$. For $i \geq 2$ define $\Omega^{i}(M)=\Omega\left(\Omega^{i-1}(M)\right)$. It can be easily proved that $\Omega^{i}(M)$ are invariant's of $M$.

2.7. Base change: Let $\phi:(A, \mathfrak{m}) \rightarrow\left(A^{\prime}, \mathfrak{m}^{\prime}\right)$ be a flat local ring homomorphism with $\mathfrak{m} A^{\prime}=\mathfrak{m}^{\prime}$. If $N$ is an $A$-module set $N^{\prime}=N \otimes A^{\prime}$. In this case it can be seen that

(1) $\lambda_{A}(N)=\lambda_{A^{\prime}}\left(N^{\prime}\right)$.

(2) $\ell_{A}\left(M / \mathfrak{m}^{n} M\right)=\ell_{A^{\prime}}\left(M^{\prime} / \mathfrak{m}^{\prime n} M^{\prime}\right)$ for all $n \geq 0$.

(3) $\operatorname{dim} M=\operatorname{dim} M^{\prime}$ and $\operatorname{depth} M=\operatorname{depth} M^{\prime}$.

(4) $e_{i}(M)=e_{i}\left(M^{\prime}\right)$ for all $i$.

(5) $\Omega_{A}(M) \otimes_{A} A^{\prime} \cong \Omega_{A^{\prime}}\left(M^{\prime}\right)$.

The specific base changes we do are the following:

(1) We can choose $A^{\prime}$ to be the completion of $A$.

(2) If $k=A / \mathfrak{m}$ is countable then we can choose $A^{\prime}$ with residue field uncountable. To do this note that by (1) we may assume $A$ is complete. Then set $A^{\prime}=A[[X]]_{\mathfrak{m} A[[X]]}$. Note that the residue field of $A^{\prime}$ is $k((X))$ which is uncountable.

2.8. A Cohen-Macaulay $A$-module $M$ is said to have minimal multiplicity if $\operatorname{deg} h_{M}(z) \leq 1$. In this case it is known that $G(M)$ is Cohen-Macaulay, see 9, Theorem 16]. If the ring $A$ has minimal multiplicity then it is easy to verify that every MCM $A$-module has minimal multiplicity.

2.9. Recall an $A$-module $U$ is said to be Ulrich if $U$ is MCM and $e(M)=\mu(M)$. It is well-known that if $U$ is Ulrich then $G(U)$ is Cohen-Macaulay. Conversely it is known that if $M$ is an MCM $A$-module with $G(M)$ Cohen-Macaulay then $M$ is Ulrich if and only if $e_{1}(M)=0$. Ulrich modules are special. Let us first recall that the following cases of CM rings when Ulrich modules are known to exist:

(1) $\operatorname{dim} A \leq 1$; (folklore; for readers convenience we prove it in 9.2).

(2) $A$ is a strict complete intersection and a quotient of a regular local ring; see $[5,2.5]$

(3) $A$ is the completion (with respect to irrelevant maximal ideal) of a two dimensional standard graded Cohen-Macaulay algebra (and a domain) over an infinite field; see [1, 4.8].

(4) If $A$ has minimal multiplicity then $\operatorname{Syz}_{1}(M)$ is Ulrich for any non-free MCM $A$-module (this is a folklore result and can be easily proved by reducing the exact sequence $0 \rightarrow \operatorname{Syz}_{1}(M) \rightarrow A^{\mu(M)} \rightarrow M \rightarrow 0$ by a maximal superficial sequence). 


\section{3. $e^{T}(M)$}

In this section we prove Theorem 1.4. This requires several preliminaries.

3.1. Let $(A \cdot m)$ be a Cohen-Macaulay local ring. Let $M \in \mathrm{CM}(A)$. In [9, Prop. 17] we proved that the function

$$
n \mapsto \ell\left(\operatorname{Tor}_{1}^{A}\left(M, \frac{A}{\mathfrak{m}^{n+1}}\right)\right)
$$

is of polynomial type, i.e., it coincides with a polynomial $t_{M}(z)$ for all $n \gg 0$. In [9. Theorem 18] we also proved that

(1) $M$ is free if and only if $\operatorname{deg} t_{M}(z)<d-1$.

(2) If $M$ is not free then $\operatorname{deg} t_{M}(z)=d-1$ and the normalized leading coefficient of $t_{M}(z)$ is $\mu(M) e_{1}(A)-e_{1}(M)-e_{1}(\Omega(M))$; here $\mu(M)$ denotes the minimal number of generators of $M$.

(3) For any $M \in \operatorname{CM}(A)$,

$$
\begin{aligned}
e_{A}^{T}(M) & =\lim _{n \rightarrow \infty} \frac{(d-1) !}{n^{d-1}} \ell\left(\operatorname{Tor}_{1}^{A}\left(M, \frac{A}{\mathfrak{m}^{n+1}}\right)\right) \\
& =\mu(M) e_{1}(A)-e_{1}(M)-e_{1}(\Omega(M)) .
\end{aligned}
$$

Remark 3.2. If $I$ is $\mathfrak{m}$-primary we may consider the function

$$
n \mapsto \ell\left(\operatorname{Tor}_{1}^{A}\left(M, \frac{A}{I^{n+1}}\right)\right) .
$$

It is easy to prove that it is of polynomial type with degree $\leq d-1$. However if $I \neq \mathfrak{m}$ then we have no control over its degree. It can be the zero polynomial even when $M$ is not free, see [9, Remark 20]. Because of these reasons our technique fails for general $\mathfrak{m}$-primary ideals.

In a previous paper [12, 2.6] we proved that if $0 \rightarrow N \rightarrow E \rightarrow M \rightarrow 0$ is an exact sequence of MCM $A$-modules then

$$
e_{A}^{T}(E) \leq e_{A}^{T}(M)+e_{A}^{T}(N) .
$$

3.3. Let $\alpha \in \operatorname{Ext}_{A}^{1}(M, N)$. Let $\alpha$ be given by an extension $0 \rightarrow N \rightarrow E \rightarrow M \rightarrow 0$. Then note that $E$ is a MCM $A$-module. Set

$$
e_{A}^{T}(\alpha)=e_{A}^{T}(M)+e_{A}^{T}(N)-e_{A}^{T}(E) .
$$

If $\alpha$ is given by an equivalent extension $0 \rightarrow N \rightarrow E^{\prime} \rightarrow M \rightarrow 0$ then $E \cong E^{\prime}$. Thus $e_{A}^{T}(\alpha)$ is well-defined. Note $e_{A}^{T}(\alpha) \geq 0$. If $\alpha=0$ then the extension is split. So $e_{A}^{T}(\alpha)=0$.

Definition 3.4. An extension $s \in \operatorname{Ext}_{A}^{1}(M, N)$ is $T$-split if $e_{A}^{T}(s)=0$.

Our next result shows that we can often reduce to dimension one.

In a previous work [12, 2.9] we proved that $e_{A}^{T}(-)$ behaves well mod superficial elements.

Proposition 3.5. Suppose $\operatorname{dim} A \geq 2$ and let $M \in \mathrm{CM}(A)$. Assume the residue field $k$ is infinite. Let $x$ be $A \oplus M \oplus \Omega_{A}(M)$-superficial. Set $B=A /(x)$ and $N=M / x M$. Then

$$
e_{B}^{T}(N)=e_{A}^{T}(M)
$$

As an easy consequence we get the following: 
Corollary 3.6. Suppose $\operatorname{dim} A \geq 2$ and let $M, N \in \mathrm{CM}(A)$. Let $s \in \operatorname{Ext}_{A}^{1}(M, N)$ be represented by an exact sequence $0 \rightarrow N \rightarrow E \rightarrow M \rightarrow 0$. Let $x$ be $A \oplus M \oplus N \oplus$ $E \oplus \Omega_{A}(M) \oplus \Omega_{A}(N) \oplus \Omega_{A}(E)$-superficial element. Set $B=A /(x)$. Then

$$
e_{A}^{T}(s)=e_{B}^{T}(s \otimes B) .
$$

Thus $s$ is $T$-split if and only if $s \otimes B$ is T-split.

In dimension one we have the following result:

Lemma 3.7. Let $(A, \mathfrak{m})$ be a Cohen-Macaulay local ring of dimension one. Let $M, N$ be $M C M$ A-modules and let $s: 0 \rightarrow N \rightarrow E \rightarrow M \rightarrow 0$ be an extension of $M$ by $N$. The following assertions are equivalent:

(i) $s$ is $T$-split.

(ii) For all $n \gg 0$ the sequence

$0 \rightarrow \operatorname{Tor}_{1}^{A}\left(A / \mathfrak{m}^{n+1}, N\right) \rightarrow \operatorname{Tor}_{1}^{A}\left(A / \mathfrak{m}^{n+1}, E\right) \rightarrow \operatorname{Tor}_{1}^{A}\left(A / \mathfrak{m}^{n+1}, M\right) \rightarrow 0$

is exact.

(iii) For all $n \gg 0$ the map $\operatorname{Tor}_{2}^{A}\left(A / \mathfrak{m}^{n+1}, E\right) \rightarrow \operatorname{Tor}_{2}^{A}\left(A / \mathfrak{m}^{n+1}, M\right)$ is surjective and the map $N / \mathfrak{m}^{n+1} N \rightarrow E / \mathfrak{m}^{n+1} E$ is injective.

Proof. As $\operatorname{dim} A=1$ we get that for any MCM $A$-module $X$ we have $e_{A}^{T}(X)=$ $\ell\left(\operatorname{Tor}_{1}^{A}\left(A / \mathfrak{m}^{n+1}, X\right)\right)$ for all $n \gg 0$. Thus (ii) $\Longrightarrow$ (i). Furthermore it is clear that (ii) and (iii) are equivalent.

We now assume (i), i.e., $s$ is $T$-split. It follows that $e_{A}^{T}(E)=e_{A}^{T}(M)+e_{A}^{T}(N)$. The exact sequence $0 \rightarrow N \rightarrow E \rightarrow M \rightarrow 0$ induces an exact sequence

$$
\rightarrow \operatorname{Tor}_{1}^{A}\left(A / \mathfrak{m}^{n+1}, N\right) \stackrel{u_{n}}{\longrightarrow} \operatorname{Tor}_{1}^{A}\left(A / \mathfrak{m}^{n+1}, E\right) \stackrel{v_{n}}{\longrightarrow} \operatorname{Tor}_{1}^{A}\left(A / \mathfrak{m}^{n+1}, M\right)
$$

For $n \gg 0$ the module in the middle has the same length as the sum of the other two. It follows that for $n \gg 0$ the map $u_{n}$ is injective and the map $v_{n}$ is surjective. The result follows.

Proposition 3.8. Let $(A, \mathfrak{m})$ be a Cohen-Macaulay local ring of dimension $d \geq$ 1 and let $M, N, N^{\prime}, E, E^{\prime}$ be $M C M$ A-modules. Suppose we have a commutative diagram

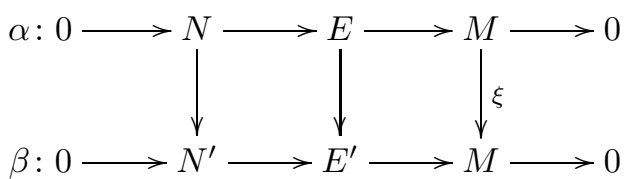

where $\xi$ is the identity map. If $\alpha$ is T-split then $\beta$ is also T-split.

Proof. By 3.6 we may assume that $\operatorname{dim} A=1$. As $\alpha$ is $T$-split we have that for $i=1,2$ the maps $u_{i, n}: \operatorname{Tor}_{i}^{A}\left(A / \mathfrak{m}^{n+1}, E\right) \rightarrow \operatorname{Tor}_{i}^{A}\left(A / \mathfrak{m}^{n+1}, M\right)$ is surjective for $i=1,2$ and for all $n \gg 0$. We also have a commutative diagram

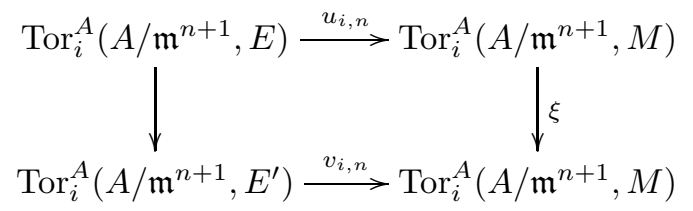

where $\xi$ is the identity map. A simple diagram chase shows that $v_{i, n}$ is also surjective for $i=1,2$ and for all $n \gg 0$. 
Proposition 3.9. Let $(A, \mathfrak{m})$ be a Cohen-Macaulay local ring of dimension $d \geq$ 1 and let $M, M^{\prime}, N, E, E^{\prime}$ be $M C M$ A-modules. Suppose we have a commutative diagram

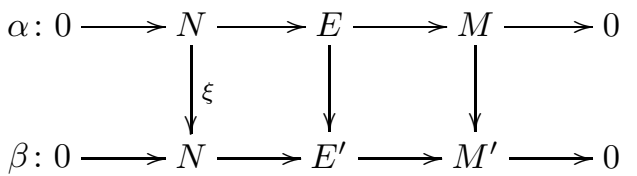

where $\xi$ is the identity map. If $\beta$ is T-split then $\alpha$ is also T-split.

Proof. By 3.6 we may assume that $\operatorname{dim} A=1$. As $\beta$ is $T$-split we have that for $i=0,1$ the maps $v_{i, n}$ : $\operatorname{Tor}_{i}^{A}\left(A / \mathfrak{m}^{n+1}, N\right) \rightarrow \operatorname{Tor}_{i}^{A}\left(A / \mathfrak{m}^{n+1}, E^{\prime}\right)$ is injective for all $n \gg 0$. We also have a commutative diagram

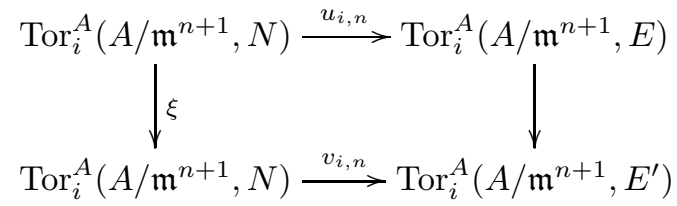

where $\xi$ is the identity map. A simple diagram chase shows that $u_{i, n}$ is also injective for $i=0,1$ and for all $n \gg 0$. The result follows.

Definition 3.10. Let $M, N$ be maximal Cohen-Macaulay $A$-modules. Set

$$
T_{A}(M, N)=\left\{s \mid s \in \operatorname{Ext}_{A}^{1}(M, N) \text { and } e_{A}^{T}(s)=0\right\} .
$$

Our next result is

Theorem 3.11. Let $(A, \mathfrak{m})$ be a Cohen-Macaulay local ring of dimension $d \geq 1$. Let $M, N$ be $M C M$ A-modules. Then $T_{A}(M, N)$ is an $A$-submodule of $\operatorname{Ext}_{1}^{A}(M, N)$.

Proof. If $\alpha$ is the split extension then $e_{A}^{T}(\alpha)=0$. So $0 \in T_{A}(M, N)$. Let $\alpha: 0 \rightarrow$ $N \rightarrow E \rightarrow M \rightarrow 0$ be an extension with $\alpha \in T_{A}(M, N)$. Then note that for $r \in A$ the extension $r \alpha$ is given by the push-out diagram:

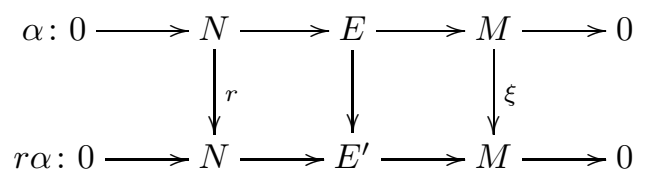

where $\xi$ is the identity map. By Proposition 3.8 we get that $r \alpha$ is also $T$-split.

Let $\alpha, \alpha^{\prime} \in T_{A}(M, N)$. Set $\alpha=0 \rightarrow N \rightarrow E \stackrel{\pi}{\rightarrow} M \rightarrow 0$ and $\alpha^{\prime}=0 \rightarrow N \rightarrow$ $E^{\prime} \stackrel{\pi^{\prime}}{\rightarrow} M \rightarrow 0$. Consider the pull-back diagram:

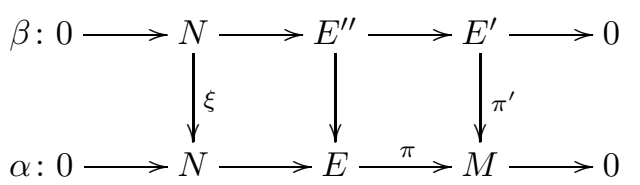

where $\xi$ is the identity map. By Proposition 3.9 we get that $\beta$ is also $T$-split. Let $\Delta=\left\{(n,-n) \in E^{\prime \prime} \mid n \in N\right\}$. Set $Y=E^{\prime \prime} / \Delta$. It is well-known that $\alpha+\alpha^{\prime}$ is given by the extension:

$$
0 \rightarrow N \rightarrow Y \stackrel{\bar{\pi}}{\rightarrow} M \rightarrow 0,
$$

where $\bar{\pi}\left(\left[e, e^{\prime}\right]\right)=\pi(e)$ (here $\left[e, e^{\prime}\right]$ is the image of $\left(e, e^{\prime}\right)$ in $\left.Y\right)$. 
We have the following commutative diagram

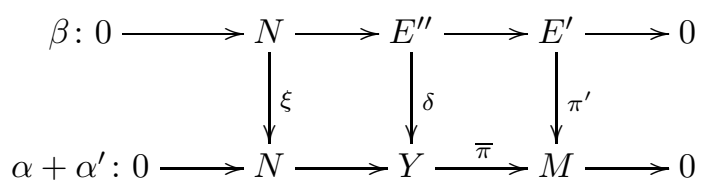

where $\xi$ is the identity map and $\delta$ is the canonical surjection. We note that $\operatorname{ker} \delta=$ $\operatorname{ker} \pi^{\prime}=N$. From the middle column we get

$$
e^{T}\left(E^{\prime \prime}\right) \leq e^{T}(Y)+e^{T}(N) .
$$

As $\alpha^{\prime}$ is $T$-split we get $e^{T}(N)=e^{T}\left(E^{\prime}\right)-e^{T}(M)$. So we get

$$
e^{T}\left(E^{\prime \prime}\right) \leq e^{T}(Y)+e^{T}\left(E^{\prime}\right)-e^{T}(M) .
$$

As $\beta T$-split we get we get $e^{T}(N)=e^{T}\left(E^{\prime}\right)-e^{T}\left(E^{\prime}\right)$. It follows that

$$
e^{T}(N) \leq e^{T}(Y)-e^{T}(M) .
$$

But the exact sequence for the extension $\alpha+\alpha^{\prime}$ yields

$$
e^{T}(N) \geq e^{T}(Y)-e^{T}(M) .
$$

It follows that $e^{T}(N)=e^{T}(Y)-e^{T}(M)$. So $\alpha+\alpha^{\prime}$ is $T$-split.

We will need the following result in the next section. The first and third assertion are well-known.

Lemma 3.12. Let $\phi:(A, \mathfrak{m}) \rightarrow\left(A^{\prime}, \mathfrak{m}^{\prime}\right)$ be a flat local ring homomorphism of Cohen-Macaulay local rings with $\mathfrak{m} A^{\prime}=\mathfrak{m}^{\prime}$. If $C$ is an $A$-module set $C^{\prime}=C \otimes A^{\prime}$. Let $M, N$ be be MCM A-modules. Then

(1) $M^{\prime}, N^{\prime}$ are $M C M A^{\prime}$-modules.

(2) $e_{A}^{T}(M)=e_{A^{\prime}}^{T}\left(M^{\prime}\right)$.

(3) If $\alpha: 0 \rightarrow N \rightarrow E \rightarrow M \rightarrow 0$ is an extension then so is

$$
\alpha^{\prime}=\alpha \otimes 1: 0 \rightarrow N^{\prime} \rightarrow E^{\prime} \rightarrow M^{\prime} \rightarrow 0 .
$$

(4) $e_{A}^{T}(\alpha)=e_{A^{\prime}}^{T}\left(\alpha^{\prime}\right)$.

(5) $\alpha \in T_{A}(M, N)$ if and only if $\alpha^{\prime} \in T_{A^{\prime}}\left(M^{\prime}, N^{\prime}\right)$.

Proof. (1) This follows from 2.7(3)

(2) This follows from $2.7(4),(5)$.

(3) This is well-known.

(4) This follows from 2.7(4), (5) and (6).

(5) This follows from (4).

We now state and prove the main result of this section.

Theorem 3.13. (with notation as above) $T_{A}: \operatorname{CM}(A) \times \operatorname{CM}(A) \rightarrow \bmod (A)$ is a sub-functor of $\operatorname{Ext}_{A}^{1}(-,-)$.

Proof. Using 3.11 it suffices to prove $T_{A}$ is a functor. Let $\alpha: 0 \rightarrow N \rightarrow E \rightarrow M \rightarrow 0$ be an extension. Let $f: N \rightarrow N^{\prime}$ be an $A$-linear map. Let $\beta: 0 \rightarrow N^{\prime} \rightarrow E^{\prime} \rightarrow$ 
$M \rightarrow 0$ be the extension corresponding to $\operatorname{Ext}_{A}^{1}(M, f)(\alpha)$. Then it is well-known (see [15, p. 207]) that we have a push-out diagram

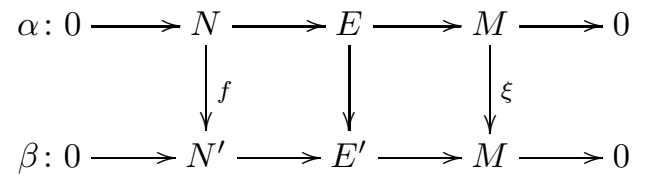

where $\xi$ is the identity map. If $\alpha$ is $T$-split then by $3.8 \beta$ is also $T$-split.

Given a map $g: M^{\prime} \rightarrow M$, let $\gamma: 0 \rightarrow N \rightarrow E^{\prime} \rightarrow M^{\prime} \rightarrow 0$ be the extension corresponding to $\operatorname{Ext}_{A}^{1}(g, N)(\alpha)$. Then it is well-known (see [15, p. 208]) that we have a pull-back diagram

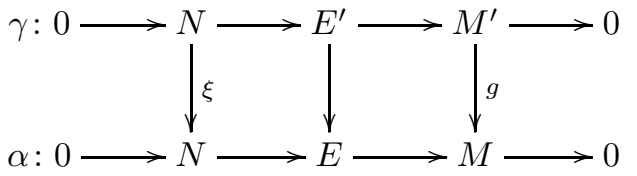

where $\xi$ is the identity map. By 3.9 it follows that $\gamma$ is also $T$-split. (note the change in labeling of modules here with respect to 3.9 ).

Thus we have shown that $T_{A}: \operatorname{CM}(A) \times \operatorname{CM}(A) \rightarrow \bmod (A)$ is a sub-functor of $\operatorname{Ext}_{A}^{1}(-,-)$.

\section{Dimension of $T_{A}(M, N)$}

In this section we prove Theorem 1.5. We re-state it for the reader's convenience.

Theorem 4.1. Let $(A, \mathfrak{m})$ be Cohen-Macaulay of dimension $d \geq 1$. Let $M, N$ be MCM A-modules. Then

$$
\operatorname{Ext}_{A}^{1}(M, N) / T_{A}(M, N) \text { has finite length. }
$$

The strategy of the proof is to prove first when $\operatorname{dim} A=1$. We will need the following preliminary results/notions.

We need the following result when $\operatorname{dim} A=1$.

Lemma 4.2. Let $(A, \mathfrak{m})$ be a Cohen-Macaulay local ring of dimension one with infinite residue field $k=A / \mathfrak{m}$. Let $M$ be a MCM A-module. Let $x \in \mathfrak{m}$ be $A$ superficial. Say $\mathfrak{m}^{c+1}=x \mathfrak{m}^{c}$. Then we have the following:

(1) Fix $n \geq c$. The map $\psi: A / \mathfrak{m}^{n} \rightarrow A / \mathfrak{m}^{n+1}$ given by $\psi\left(a+\mathfrak{m}^{n}\right)=x a+\mathfrak{m}^{n+1}$ induces an isomorphism $\operatorname{Tor}_{1}^{A}\left(M, A / \mathfrak{m}^{n}\right) \cong \operatorname{Tor}_{1}^{A}\left(M, A / \mathfrak{m}^{n+1}\right)$.

(2) $e^{T}(M)=\ell\left(\operatorname{Tor}_{1}^{A}\left(M, A / \mathfrak{m}^{n+1}\right)\right)$ for all $n \geq c$.

Proof. (1) We have the following exact sequence

$$
0 \rightarrow \frac{\left(\mathfrak{m}^{n+1}: x\right)}{\mathfrak{m}^{n}} \rightarrow A / \mathfrak{m}^{n} \stackrel{\psi}{\rightarrow} A / \mathfrak{m}^{n+1} \rightarrow A /\left(\mathfrak{m}^{n+1}, x\right) \rightarrow 0 .
$$

As $A$ is Cohen-Macaulay we get that $x$ is $A$-regular. Furthermore as $\mathfrak{m}^{c+1}=x \mathfrak{m}^{c}$ and $n \geq c$ we get that $\left(\mathfrak{m}^{n+1}: x\right)=\mathfrak{m}^{n}$. Furthermore we have $\mathfrak{m}^{n+1} \subseteq(x)$. So $A /\left(\mathfrak{m}^{n+1}, x\right)=A /(x)$. Thus the above exact sequence reduces to

$$
0 \rightarrow A / \mathfrak{m}^{n} \stackrel{\psi}{\rightarrow} A / \mathfrak{m}^{n+1} \rightarrow A /(x) \rightarrow 0 .
$$


As $M$ is an MCM $A$-module we get that $x$ is also $M$-regular. Thus $\operatorname{Tor}_{i}^{A}(M, A /(x))=0$ for $i \geq 1$. Applying the functor $M \otimes-$ we get the required result.

(2) By definition we get that $e^{T}(M)=\ell\left(\operatorname{Tor}_{1}^{A}\left(M, A / \mathfrak{m}^{n+1}\right)\right)$ for all $n \gg 0$. By (1) we get the required result.

The following result is a basic ingredient to prove Theorem 4.1 when $\operatorname{dim} A=1$.

Lemma 4.3. Let $(A, \mathfrak{m})$ be a Cohen-Macaulay local ring of dimension one with infinite residue field $k=A / \mathfrak{m}$. Let $x \in \mathfrak{m}$ be $A$-superficial. Say $\mathfrak{m}^{c+1}=x \mathfrak{m}^{c}$. Let $M, N$ be $M C M A$-modules and let $\alpha \in \operatorname{Ext}_{A}^{1}(M, N)$. Let $u \in \mathfrak{m}^{n}$ where $n \geq c+1$. Then

(1) $e^{T}(u \alpha) \leq e^{T}(\alpha)$.

(2) $e^{T}(u \alpha)=e^{T}(\alpha)$ if and only if $\alpha$ is T-split.

4.4. Before proving Lemma 4.3 let us prove Theorem 4.1 when $\operatorname{dim} A=1$ and $A$ contains an infinite residue field.

Proof. (with setup as in Lemma 4.3). Let $\alpha \in \operatorname{Ext}_{A}^{1}(M, N)$. Let $u \in \mathfrak{m}^{c+1}$ be $A$-regular. Then by Lemma 4.3 for all $i \geq 1$ we have

$$
0 \leq \cdots \leq e^{T}\left(u^{i+1} \alpha\right) \leq e^{T}\left(u^{i} \alpha\right) \leq \cdots \leq e^{T}(u \alpha) \leq e^{T}(\alpha)
$$

It follows that there exists $i_{0}$ such that $e^{T}\left(u^{i+1} \alpha\right)=e^{T}\left(u^{i} \alpha\right)$ for all $i \geq i_{0}$. It follows from Lemma 4.3 that $u^{i} \alpha$ is $T$-split all $i \geq i_{0}$.

Now let $\alpha_{1}, \ldots, \alpha_{s}$ generate $\operatorname{Ext}_{A}^{1}(M, N)$ as an $A$-module. By the previous argument we get that there exists $l$ such that $u^{i} \alpha_{j}$ is $T$-split for all $i \geq l$ and for $j=1, \ldots, s$. So $u^{l} \operatorname{Ext}_{A}^{1}(M, N) \subseteq T_{A}(M, N)$. As $u^{l}$ is $A$-regular the result follows.

We now give

Proof of Lemma 4.3. Let $\alpha: 0 \rightarrow N \rightarrow C \rightarrow M \rightarrow 0$. Then note that for $u \in \mathfrak{m}^{n+1}$ the extension $u \alpha$ is given by the push-out diagram:

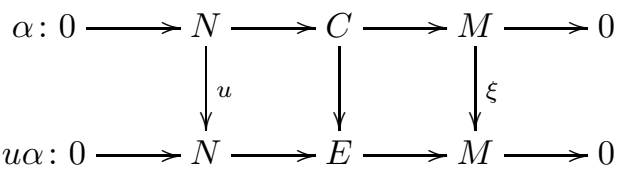

where $\xi$ is the identity map. It suffices to prove the following assertions

(i) $e^{T}(E) \geq e^{T}(C)$.

(ii) $e^{T}(E)=e^{T}(C)$ if and only if $\alpha$ is $T$-split.

4.5. Tensoring the above commutative diagram with $A / \mathfrak{m}^{n}$ we obtain a commutative diagram

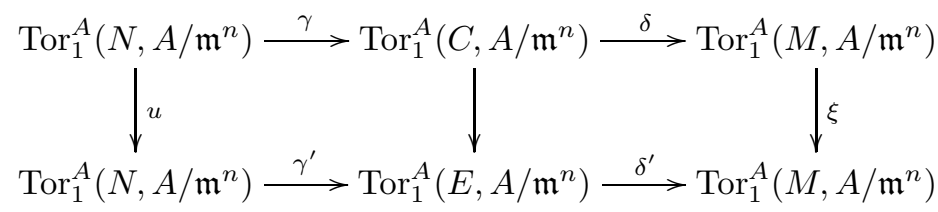

Here $\xi$ is the identity map. We note that as $u \in \mathfrak{m}^{n}$ we get that the multiplication map on $\operatorname{Tor}_{1}^{A}\left(N, A / \mathfrak{m}^{n}\right)$ by $u$ is the zero map. Set $U=$ image $\gamma$ and $U^{\prime}=$ image $\gamma^{\prime}$. Also set $V=$ image $\delta$ and $V^{\prime}=$ image $\delta^{\prime}$. 
4.6. We have a commutative diagram

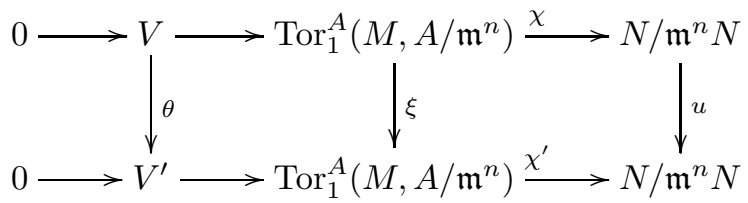

where $\theta$ is induced by the maps $\xi$ and multiplication by $u$ (note $\xi$ is the identity map). Thus $\theta$ is injective and so $\ell(V) \leq \ell\left(V^{\prime}\right)$.

A similar argument as above yields a natural map $\eta: U \rightarrow U^{\prime}$ which is the zero map as the multiplication map on $\operatorname{Tor}_{1}^{A}\left(N, A / \mathfrak{m}^{n}\right)$ by $u$ is the zero map.

4.7. We have a commutative diagram

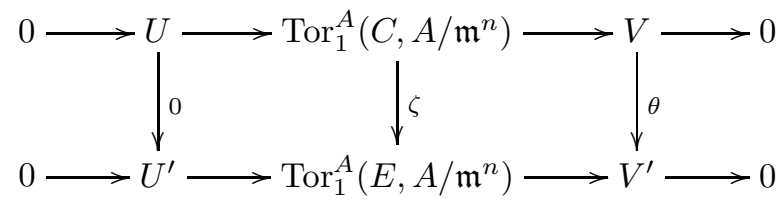

Note that as $\theta$ is injective we have that $\operatorname{ker} \zeta \cong U$. Set $\operatorname{coker} \zeta=W$. We get

$$
\begin{aligned}
e^{T}(E)-e^{T}(C) & =\ell\left(\operatorname{Tor}_{1}^{A}\left(E, A / \mathfrak{m}^{n}\right)\right)-\ell\left(\operatorname{Tor}_{1}^{A}\left(C, A / \mathfrak{m}^{n}\right)\right) \quad \text { by Lemma } 4.2(2) \\
& =\ell(W)-\ell(U) \\
& =\ell\left(U^{\prime}\right)+\ell\left(V^{\prime} / V\right)-\ell(U) .
\end{aligned}
$$

We get the last quality by using the Snake Lemma on the commutative diagram 4.7.

We now assert that $\ell(U) \leq \ell\left(U^{\prime}\right)$. To see this consider the following commutative diagram

\section{8 .}

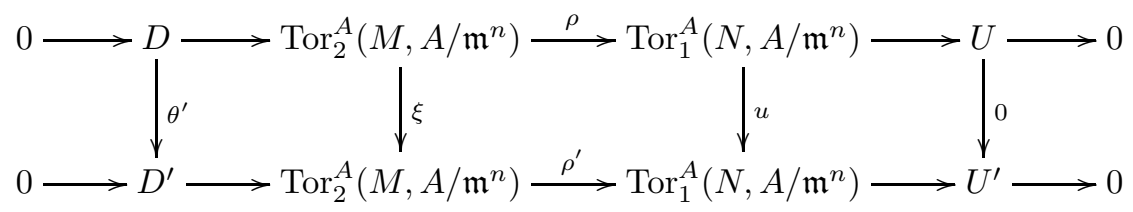

(here $\xi$ is the identity map) and $\theta^{\prime}: D \rightarrow D^{\prime}$ is the map induced by $\xi$ and multiplication map on $\operatorname{Tor}_{1}^{A}\left(N, A / \mathfrak{m}^{n}\right)$ by $u$. Clearly $\theta^{\prime}$ is injective. Thus $\ell(D) \leq \ell\left(D^{\prime}\right)$. We also have

(1) $\ell(U)=\ell\left(\operatorname{Tor}_{1}^{A}\left(N, A / \mathfrak{m}^{n}\right)\right)-\ell\left(\operatorname{Tor}_{2}^{A}\left(M, A / \mathfrak{m}^{n}\right)\right)+\ell(D)$.

(2) $\ell\left(U^{\prime}\right)=\ell\left(\operatorname{Tor}_{1}^{A}\left(N, A / \mathfrak{m}^{n}\right)\right)-\ell\left(\operatorname{Tor}_{2}^{A}\left(M, A / \mathfrak{m}^{n}\right)\right)+\ell\left(D^{\prime}\right)$.

4.9. It follows that $\ell(U) \leq \ell\left(U^{\prime}\right)$ with equality if and only if $\theta^{\prime}: D \rightarrow D^{\prime}$ is an isomorphism.

We now prove our assertions. (i) $e^{T}(E) \geq e^{T}(C)$. This follows from 4.7 .3 and 4.9 .

(ii) If $\alpha$ is $T$-split then so is $u \alpha$. As $\alpha$ is $T$-split it follows that $e^{T}(M)+e^{T}(N)-$ $e^{T}(C)=0$. As As $u \alpha$ is $T$-split it follows that $e^{T}(M)+e^{T}(N)-e^{T}(E)=0$. So $e^{T}(E)=e^{T}(C)$. 
Conversely if $e^{T}(E)=e^{T}(C)$ we get by 4.7 .3 and 4.9 that $\ell(V)=\ell\left(V^{\prime}\right)$ and $\ell(U)=\ell\left(U^{\prime}\right)$. We also get that $\theta: V \rightarrow V^{\prime}$ and $\theta^{\prime}: D \rightarrow D^{\prime}$ are isomorphisms.

In 4.8 let $X=$ image $\rho$ and let $X^{\prime}=$ image $\rho^{\prime}$. Furthermore let $\kappa: X \rightarrow X^{\prime}$ be the map induced by $\theta^{\prime}$ and $\xi$. As $\theta^{\prime}$ and $\xi$ are isomorphism's we get that $\kappa$ is also an isomorphism. By 4.8 we also get the following commutative diagram:

4.10.

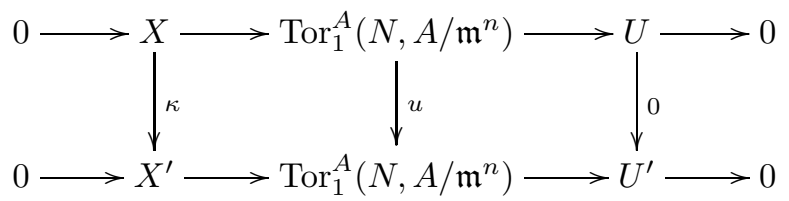

As $u \in \mathfrak{m}^{n}$ the multiplication map on $\operatorname{Tor}_{1}^{A}\left(N, A / \mathfrak{m}^{n}\right)$ by $u$ is zero. It follows that $\kappa$ is the zero map. But $\kappa$ is also an isomorphism. So $X=X^{\prime}=0$. Thus the map $\operatorname{Tor}_{1}^{A}\left(N, A / \mathfrak{m}^{n}\right) \rightarrow U$ is an isomorphism.

We now consider the commutative diagram 4.6, Let $Y=$ image $\chi$ and $Y^{\prime}=$ image $\chi^{\prime}$. We note that as $\theta, \xi$ are isomorphism's the induced map $\kappa^{\prime}: Y \rightarrow Y^{\prime}$ is an isomorphism. But we also have a commutative diagram

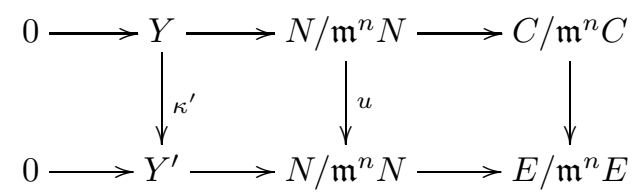

As $u \in \mathfrak{m}^{n}$ the multiplication map on $N / \mathfrak{m}^{n} N$ by $u$ is the zero map. So $\kappa^{\prime}$ is the zero map. But as argued before $\kappa^{\prime}$ is an isomorphism. It follows that $Y=Y^{\prime}=0$. So by 4.6 we get that $V \cong \operatorname{Tor}_{1}^{A}\left(M, A / \mathfrak{m}^{n}\right)$. Thus we have an exact sequence

$$
0 \rightarrow \operatorname{Tor}_{1}^{A}\left(N, A / \mathfrak{m}^{n}\right) \rightarrow \operatorname{Tor}_{1}^{A}\left(C, A / \mathfrak{m}^{n}\right) \rightarrow \operatorname{Tor}_{1}^{A}\left(M, A / \mathfrak{m}^{n}\right) \rightarrow 0 .
$$

By 4.2 we get that $e^{T}(\alpha)=0$.

We now give

Proof of Theorem 4.1. We consider two cases.

Case-1: The residue field $k=A / \mathfrak{m}$ is uncountable.

We prove the result by induction on $d=\operatorname{dim} A$. When $d=1$ the result follows from 4.4. We now assume $d \geq 2$ and the results is known for Cohen-Macaulay local rings with uncountable residue field and dimension $d-1$. Let $\alpha: 0 \rightarrow N \rightarrow C \rightarrow$ $M \rightarrow 0$ and let $a \in \mathfrak{m}$. Then for every $n \geq 1$ the extension $a^{n} \alpha$ is given by the push-out diagram:

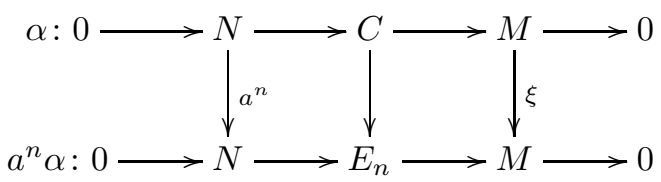

where $\xi$ is the identity map. As $k$ is uncountable we can choose $x \in \mathfrak{m}$ such that for all $n \geq 1$

$$
x \text { is } A \oplus M \oplus N \oplus C \oplus E_{n} \oplus \Omega_{A}(M) \oplus \Omega_{A}(N) \oplus \Omega_{A}(C) \oplus \Omega_{A}\left(E_{n}\right) \text { superficial }
$$

(see for instance [13, 2.2])). Set $B=A /(x)$. Set $\bar{a}$ to be image of $a$ in $B$. By 3.6 we get that

$$
e_{A}^{T}\left(a^{n} \alpha\right)=e_{B}^{T}\left(\left(a^{n} \alpha\right) \otimes B\right)=e_{B}^{T}\left(\bar{a}^{n}(\alpha \otimes B)\right) .
$$


By our induction hypothesis we have $e_{B}^{T}\left(\bar{a}^{n}(\alpha \otimes B)=0\right.$ for $n \gg 0$. Therefore we have $e_{A}^{T}\left(a^{n} \alpha\right)=0$ for $n \gg 0$. Thus $a^{n} \alpha \in T_{A}(M, N)$ for $n \gg 0$. It then can be easily checked that there exists $n$ such that $a^{n} \operatorname{Ext}_{A}^{1}(M, N) \subseteq T(M, N)$ for all $n \gg 0$. Let $\mathfrak{m}$ be generated by $a_{1}, \ldots, a_{r}$. By our previous argument we get $n_{i}$ such that $a_{i}^{n_{i}} \operatorname{Ext}_{A}^{1}(M, N) \subseteq T(M, N)$. It follows that

$$
\left(a_{1}^{n_{1}}, \cdots, a_{r}^{n_{r}}\right) \operatorname{Ext}_{A}^{1}(M, N) \subseteq T(M, N) .
$$

As $\left(a_{1}^{n_{1}}, \cdots, a_{r}^{n_{r}}\right)$ is $\mathfrak{m}$-primary the result follows.

Case 2: The residue field $k$ of $A$ is either finite or countably infinite,

We choose a local flat extension $(B, \mathfrak{n})$ with $\mathfrak{m} B=\mathfrak{n}$ such that the residue field $l=B / \mathfrak{n}$ is uncountable. By 3.12 we have that $\xi \in T_{A}(M, N)$ if and only if $\xi \otimes 1 \in T_{B}(M \otimes B, N \otimes B)$. Let $\alpha \in \operatorname{Ext}_{A}^{1}(M, N)$ and let $a \in \mathfrak{m}$. Then for all $n \geq 1$ we have

$$
e_{A}^{T}\left(a^{n} \alpha\right)=e_{B}^{T}\left(\left(a^{n} \alpha\right) \otimes B\right)=e_{B}^{T}\left((a \otimes 1)^{n}(\alpha \otimes B)\right) .
$$

By our Case 1 it follows that $e_{B}^{T}\left((a \otimes 1)^{n}(\alpha \otimes B)\right)=0$ for $n \gg 0$. So $e_{A}^{T}\left(a^{n} \alpha\right)=0$ for $n \gg 0$. Thus $a^{n} \alpha \in T_{A}(M, N)$ for $n \gg 0$. By an argument similar to case 1 we get that $\ell\left(\operatorname{Ext}_{A}^{1}(M, N) / T_{A}(M, N)\right)$ is finite.

\section{Some Properties of $L^{I}(M)$}

Throughout this section $(A, \mathfrak{m})$ is a Noetherian local ring, $M$ is a Cohen-Macaulay module of dimension $r \geq 1$ and $I$ is an ideal of definition for $M$ (i.e., $\ell(M / I M)$ is finite). Set $\mathcal{R}=A[I t]$; the Rees Algebra of $I$. In [11, 4.2] we proved that $L^{I}(M)=\bigoplus_{n \geq 0} M / I^{n} M$ is a $\mathcal{R}$-module. Note that $L^{I}(M)$ is not finitely generated as a $\mathcal{R}$-module. In this section we recall an collect few of properties of $L^{I}(M)$ which we proved in [11. We also prove a result on associate primes of $L^{I}(M)$ that we need in this paper.

5.1. Set $\mathcal{M}=\mathfrak{m} \oplus \mathcal{R}(I)_{+}$. Let $H^{i}(-)=H_{\mathcal{M}}^{i}$ denote the $i^{\text {th }}$-local cohomology functor with respect to $\mathcal{M}$. Recall a graded $\mathcal{R}$-module $N$ is said to be ${ }^{*}$-Artinian if every descending chain of graded submodules of $N$ terminates. For example if $E$ is a finitely generated $\mathcal{R}$-module then $H^{i}(E)$ is *-Artinian for all $i \geq 0$.

5.2. In [11, 4.7] we proved that

$$
H^{0}\left(L^{I}(M)\right)=\bigoplus_{n \geq 0} \frac{\widetilde{I^{n+1} M}}{I^{n+1} M} .
$$

5.3. For $L^{I}(M)$ we proved that for $0 \leq i \leq r-1$

(a) $H^{i}\left(L^{I}(M)\right)$ are *-Artinian; see [11, 4.4].

(b) $H^{i}\left(L^{I}(M)\right)_{n}=0$ for all $n \gg 0$; see [11, 1.10$]$.

(c) $H^{i}\left(L^{I}(M)\right)_{n}$ has finite length for all $n \in \mathbb{Z}$; see [11, 6.4].

(d) $\lambda\left(H^{i}\left(L^{I}(M)\right)_{n}\right)$ coincides with a polynomial for all $n \ll 0$; see [11, 6.4].

5.4. The natural maps $0 \rightarrow I^{n} M / I^{n+1} M \rightarrow M / I^{n+1} M \rightarrow M / I^{n} M \rightarrow 0$ induce an exact sequence of $R(I)$-modules

$$
0 \longrightarrow G_{I}(M) \longrightarrow L^{I}(M) \stackrel{\Pi_{M}}{\longrightarrow} L^{I}(M)(-1) \longrightarrow 0 .
$$

We call (5.4.4) the first fundamental exact sequence. We use (5.4.4) also to relate the local cohomology of $G_{I}(M)$ and $L^{I}(M)$. 
5.5. An easy consequence of 5.3 and 5.4 is that

$$
H^{i}\left(G_{I}(M)\right)=0 \text { for } i=0,1, \ldots, s \quad \text { iff } \quad H^{i}\left(L^{I}(M)\right)=0 \text { for } i=0,1, \ldots, s .
$$

We need the following result in this paper.

Proposition 5.6. Assume $\operatorname{depth} A \oplus M>0$. We have

$$
\operatorname{Ass}_{\mathcal{R}(I)} G_{I}(M)=\operatorname{Ass}_{\mathcal{R}(I)} L^{I}(M) .
$$

Proof. Set $\mathcal{R}=\mathcal{R}(I), G=G_{I}(M)$ and $L=L^{I}(M)$. Let $L_{r}$ be $\mathcal{R}$-submodule of $L$ defined as follows:

$$
L_{r}=\left\langle\bigoplus_{n=0}^{r} \frac{M}{I^{n+1} M}\right\rangle .
$$

Then $L_{r}$ is a finitely generated $\mathcal{R}$-module. We note that $L_{r} \subseteq L_{r+1}$ for all $r \geq 0$ and $L=\bigcup_{r \geq 0} L_{r}$. So Ass $L=\bigcup_{r \geq 0}$ Ass $L_{r}$. Note that $L_{0}=G$. So Ass $G \subseteq \operatorname{Ass} L$.

For all $r \geq 0$ we have an exact sequence

$$
0 \rightarrow L_{r-1} \rightarrow L_{r} \stackrel{\rho_{r}}{\longrightarrow} G(-r) \rightarrow 0 .
$$

For $r=1$ we get

$$
0 \rightarrow G \rightarrow L_{1} \rightarrow G(-1) \rightarrow 0 .
$$

So we have Ass $L_{1} \subseteq \operatorname{Ass} G$. Iterating from (5.6.5) we get Ass $L_{r} \subseteq \operatorname{Ass} G$ for all $r \geq 1$. The result follows.

\section{BASIC LEMMA}

6.1. Throughout this section $(A, \mathfrak{m})$ is a Cohen-Macaulay local ring of dimension $d \geq 1, \mathcal{R}=\mathcal{R}(\mathfrak{m})$ the Rees algebra of $A$ with respect to $\mathfrak{m}$. Throughout if $M$ is an $A$-module then set $G(M)=G_{\mathfrak{m}}(M)$ and $L(M)=L^{\mathfrak{m}}(M)$. In this section we prove Lemma 1.7 It is convenient to prove a slightly more general version, see 6.3.

6.2. For $i \geq 1$ set

$$
L_{i}(M)=\operatorname{Tor}_{i}^{A}\left(M, L_{0}(A)\right)=\bigoplus_{n \geq 0} \operatorname{Tor}_{i}^{A}\left(M, A / \mathfrak{m}^{n+1}\right) .
$$

We assert that $L_{i}(M)$ is a finitely generated $\mathcal{R}$-module for $i \geq 1$. It is sufficient to prove it for $i=1$. We tensor the exact sequence $0 \rightarrow \mathcal{R} \rightarrow A[t] \rightarrow L_{0}(A)(-1) \rightarrow 0$ with $M$ to obtain a sequence of $\mathcal{R}$-modules

$$
0 \rightarrow L_{1}(M)(-1) \rightarrow \mathcal{R} \otimes_{A} M \rightarrow M[t] \rightarrow L_{0}(M)(-1) \rightarrow 0 .
$$

Thus $L_{1}(M)(-1)$ is a $\mathcal{R}$-submodule of $\mathcal{R} \otimes_{A} M$. The latter module is a finitely generated $\mathcal{R}$-module. It follows that $L_{1}(M)$ is a finitely generated $\mathcal{R}$-module.

Next we show:

Lemma 6.3. (with assumptions as in 6.1.) Further assume that $M, N, E$ are $M C M$ $A$-modules and we have a T-split exact sequence $s: 0 \rightarrow N \rightarrow E \rightarrow M \rightarrow 0$. Assume that $G(N)$ is Cohen-Macaulay. Then we have short exact sequence

$$
0 \rightarrow L(N) \rightarrow L(E) \rightarrow L(M) \rightarrow 0 ;
$$

and hence a short-exact sequence

$$
0 \rightarrow G(N) \rightarrow G(E) \rightarrow G(M) \rightarrow 0 .
$$

Furthermore $e_{i}(E)=e_{i}(N)+e_{i}(M)$ for $i=0,1, \ldots, d$. 
Proof. It is clear that $e_{0}(E)=e_{0}(N)+e_{0}(M)$. We prove rest of the assertion by induction on dimension $d \geq 1$.

We first consider the case $d=1$. As $s$ is $T$-split we have that

$$
0 \rightarrow L_{1}(N)_{n} \rightarrow L_{1}(E)_{n} \rightarrow L_{1}(M)_{n} \rightarrow 0
$$

is exact for $n \gg 0$, see 3.7. It follows we have an exact sequence of $\mathcal{R}$-modules

$$
0 \rightarrow K \rightarrow L(N) \rightarrow L(E) \rightarrow L(N) \rightarrow 0
$$

where $\lambda(K)<\infty$. As $H_{\mathcal{M}}^{0}(L(N))=0$ (since $H_{\mathcal{M}}^{0}(G(N))=0$ ) we get that $K=0$. Thus we have an exact sequence $0 \rightarrow L(N) \rightarrow L(E) \rightarrow L(M) \rightarrow 0$. In particular we have $e_{1}(E)=e_{1}(M)+e_{1}(N)$.

Now assume that $d \geq 2$ and the result has been proved for all Cohen-Macaulay local rings and all $T$-split sequences satisfying our hypothesis. By 3.6 it follows that if $x \in \mathfrak{m}$ is sufficiently general then $s \otimes A /(x)$ is $T$-split. Set $\overline{(-)}=(-) \otimes(A /(x))$. So we get that $e_{i}(\bar{E})=e_{i}(\bar{M})+e_{i}(\bar{N})$ for $i=0, \ldots, d-1$. By our choice of $x$ we get that $e_{i}(E)=e_{i}(N)+e_{i}(M)$ for $i=0,1, \ldots, d-1$. Tensoring $s$ with $L(A)$ we get sequence of $\mathcal{R}$-modules

$$
L_{1}(E) \rightarrow L_{1}(M) \stackrel{\delta}{\rightarrow} L(N) \rightarrow L(E) \rightarrow L(M) \rightarrow 0 .
$$

Set $K=$ image $\delta$. As $L_{1}(M)$ is finitely generated as a $\mathcal{R}$-module we get that $K$ is finitely generated as an $\mathcal{R}$-module. We have exact sequence

$$
0 \rightarrow K \rightarrow L(N) \rightarrow L(E) \rightarrow L(M) \rightarrow 0 .
$$

As $e_{i}(E)=e_{i}(N)+e_{i}(M)$ for $i=0,1, \ldots, d-1$ we get $\operatorname{dim} K \leq 1$ as a $\mathcal{R}$-module. Suppose if possible $K \neq 0$. Let $P \in \operatorname{Min} K$. Then $P \in \operatorname{Ass} L(N)=\operatorname{Ass}_{\mathcal{R}} G(N)$ (by 5.6). But $G(N)$ is Cohen-Macaulay $\mathcal{R}$-module of dimension $d$. So height $P=1$. This implies that $\operatorname{dim} K \geq d$. But $d \geq 2$ and we have shown that $\operatorname{dim} K \leq 1$. It follows that $K=0$. Thus we have an exact sequence $0 \rightarrow L(N) \rightarrow L(E) \rightarrow$ $L(M) \rightarrow 0$. In particular we have $e_{i}(E)=e_{i}(M)+e_{i}(N)$ for $i=0, \ldots, d$.

To prove the result regarding associated graded modules, note that we have the following commutative diagram

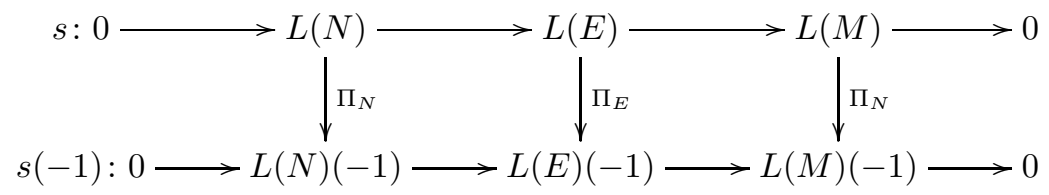

The result follows by applying (5.4.4) and the Snake Lemma.

As an immediate corollary we get

Corollary 6.4. (with hypotheses as in 6.3.) Further assume that $G(M)$ is also Cohen-Macaulay. Then $G(E)$ is Cohen-Macaulay.

\section{Proof of Theorem 1.8}

In this section we give a proof of Theorem 1.8, We restate it here for the reader's convenience. We also indicate two other results whose proofs are parallel to our main result. 
Theorem 7.1. Let $(A, \mathfrak{m})$ be a Henselian Cohen-Macaulay local ring of dimension $d \geq 1$. Suppose $M, N$ are MCM modules with $G(M), G(N)$ Cohen-Macaulay. If there exists only finitely many non-isomorphic MCM A-modules $D$ with $G(D)$ Cohen-Macaulay and $e(D)=e(M)+e(N)$; then $T_{A}(M, N)$ has finite length (in particular $\operatorname{Ext}_{A}^{1}(M, N)$ has finite length). If $h$ is the number of such isomorphism classes then $\mathfrak{m}^{h}$ annihilates $T_{A}(M, N)$.

Remark 7.2. Before we prove Theorem 7.1 note that it is formally similar to the statement of a result by Huneke and Leuschke [6, Theorem 1]. The proof is similar too except in one detail which we describe.

Proof. Let $\chi \in T_{A}(M, N)$. Let $r_{1}, \ldots, r_{h} \in \mathfrak{m}$. We prove $r_{1} \cdots r_{h} \chi=0$. Let

$$
\chi: 0 \rightarrow N \rightarrow K \rightarrow M \rightarrow 0 .
$$

Consider

$$
r_{1} \cdots r_{n} \chi: 0 \rightarrow N \rightarrow K_{n} \rightarrow M \rightarrow 0
$$

where $n$ runs through all positive integers and each $r_{i} \in \mathfrak{m}$. First note that $K_{n}$ is a MCM $A$-module for all $n \geq 1$. Further note that as $T_{A}(M, N)$ is an $A$-submodule of $\operatorname{Ext}_{A}^{1}(M, N)$ we get $r_{1} \cdots r_{n} \chi$ is $T$-split. In particular by 6.4 we get that $G\left(K_{n}\right)$ is Cohen-Macaulay for all $n \geq 1$. Furthermore notice $e\left(K_{n}\right)=e(M)+e(N)$. By our assumption there must be repetitions among the $K_{n}$. The rest of the proof is similar to [6, Theorem 1].

The following two results have proofs similar to Theorem 7.1 .

Theorem 7.3. Let $(A, \mathfrak{m})$ be a Henselian Cohen-Macaulay local ring of dimension $d \geq 1$. Suppose $M, N$ are Ulrich modules. If there exists only finitely many nonisomorphic Ulrich A-modules $D$ with $e(D)=e(M)+e(N)$; then $T_{A}(M, N)$ has finite length (in particular $\operatorname{Ext}_{A}^{1}(M, N)$ has finite length). If $h$ is the number of such isomorphism classes then $\mathfrak{m}^{h}$ annihilates $T_{A}(M, N)$.

Proof. We only note that if $\chi: 0 \rightarrow N \rightarrow E \rightarrow M \rightarrow 0$ is in $T_{A}(M, N)$ and both $M$ and $N$ Ulrich then $E$ is MCM and $G(E)$ is Cohen-Macaulay. By 6.3 $e_{1}(E)=e_{1}(M)+e_{1}(N)=0$. So $E$ is Ulrich. The rest of the proof is similar to proof of Theorem 7.1 .

Our next result is

Theorem 7.4. Let $(A, \mathfrak{m})$ be a Henselian Cohen-Macaulay local ring of dimension $d \geq 1$. Suppose $M, N$ are MCM modules with minimal multiplicity. If there exists only finitely many non-isomorphic MCM A-modules $D$ having minimal multiplicity and $e(D)=e(M)+e(N)$; then $T_{A}(M, N)$ has finite length (in particular $\operatorname{Ext}_{A}^{1}(M, N)$ has finite length). If $h$ is the number of such isomorphism classes then $\mathfrak{m}^{h}$ annihilates $T_{A}(M, N)$.

Proof. We first note that as $M, N$ have minimal multiplicity both $G(M), G(N)$ are Cohen-Macaulay. Also note that if $\chi: 0 \rightarrow N \rightarrow E \rightarrow M \rightarrow 0$ is in $T_{A}(M, N)$ and both $M$ and $N$ having minimal multiplicity. then $E$ is MCM and $G(E)$ is Cohen-Macaulay. Note $e_{2}(E)=e_{2}(M)+e_{2}(N)=0$ see 6.3. So $E$ has minimal multiplicity. The rest of the proof is similar to proof of Theorem 7.1 . 


\section{Strict COMPlete intersections}

In this section we prove the following result.

Theorem 8.1. Let $(Q, \mathfrak{n})$ be a Henselian regular local ring and let $A=Q /\left(f_{1}, \ldots, f_{c}\right)$ be a strict complete intersection. Let $f_{1}=g^{i} h$ with $g$ irreducible, ( $h$ is possibly a unit if $i \geq 2$ and is a non-unit if $i=1$ ) and $g$ does not divide $h$. If $i \geq 2$ assume $\operatorname{dim} A \geq 1$. If $i=1$ assume $\operatorname{dim} A \geq 2$. Then there exists $\left\{E_{n}\right\}_{n \geq 1}$ indecomposable MCM A-modules with bounded multiplicity and having $G\left(E_{n}\right)$ Cohen-Macaulay for all $n \geq 1$.

The proof follows by first analyzing $Q /\left(f_{1}\right)$. The following result is easy to prove.

Proposition 8.2. Let $(Q, \mathfrak{n})$ be a Henselian regular local ring and let $A=Q /(f)$ with $f \in n^{2}$. Let $f=g^{i} h$ where $g$ is irreducible ( $h$ is possibly a unit if $i \geq 2$ and is a non-unit if $i=1)$ and $g$ does not divide $h$. Then

(1) $A /(g)=Q /(g)$ and $A /\left(g^{i-1} h\right)=Q /\left(g^{i-1} h\right)$.

(2) $g A$ is a prime ideal in $A$ of height zero.

(3) $A /(g)$ and $A /\left(g^{i-1} h\right)$ are $M C M$ A-modules with Cohen-Macaulay associated graded modules.

(4) $g A \cong Q /\left(g^{i-1} h\right)$ and $\left(g^{i-1} h\right) A \cong Q /(g)$.

(5) The following is a minimal periodic free resolution of $A /(g)$.

$$
\cdots \rightarrow A \stackrel{g^{i-1} h}{\longrightarrow} A \stackrel{g}{\rightarrow} A \rightarrow 0 .
$$

We now give an estimate of dimension of a certain Ext module.

Proposition 8.3. (with hypotheses as in 8.2 Let $D=\operatorname{Ext}_{A}^{1}\left(A /(g), A /\left(g^{i-1} h\right)\right.$ ). If $i \geq 2$ then $\operatorname{dim} D=\operatorname{dim} A$. If $i=1$ then $\operatorname{dim} D \geq \operatorname{dim} A-1$.

Proof. We first consider the case $i \geq 2$. Consider the short exact sequence

$$
s: 0 \rightarrow A /\left(g^{i-1} h\right) \rightarrow A \rightarrow A /(g) \rightarrow 0 .
$$

Let $P=g A$ a prime ideal of height zero in $A$. If $s_{P}=0$ then note that $\kappa(P)=$ $(A /(g))_{P}$ the residue field of $P$ is a free $A_{P}$-module. This implies that $A_{P}$ is regular. However note that $A_{P}=Q_{(g)} /\left(g^{i}\right)$ has nilpotent elements, a contradiction. So $s_{P} \neq 0$. The result follows.

We now consider the case $i=1$. Let $\beta$ be a prime ideal of height two in $Q$ minimal over $g, h$. Then $\mathfrak{q}=\beta /(f)$ is a height one prime ideal in $A$. Consider the short exact sequence

$$
s: 0 \rightarrow A /(h) \rightarrow A \rightarrow A /(g) \rightarrow 0
$$

Note $(A /(h))_{\mathfrak{q}}$ and $(A /(g))_{\mathfrak{q}}$ are non-zero. Therefore $s_{\mathfrak{q}} \neq 0$. The result follows.

We now give a proof of Theorem 8.1 in the case of hypersurfaces.

Proof. We note that both $G(A /(g))$ and $G\left(A /\left(g^{i-1} h\right)\right)$ are Cohen-Macaulay. By Proposition 8.3 under our assumptions we have $\operatorname{dim} \operatorname{Ext}_{A}^{1}\left(A /(g), A /\left(g^{i-1} h\right)\right) \geq 1$. By Theorem 7.1 the result follows.

To prove Theorem 8.1 in general we need the next two results which are certainly known to experts. However we give proofs as we cannot find a reference. Before stating the result we make the convention that dimension of the zero module is -1 . 
Lemma 8.4. Let $(A, \mathfrak{m})$ be a Cohen-Macaulay local ring and let $E$ be a finitely generated $A$-module with $\operatorname{dim} E=r \geq 1$. Let $x \in \mathfrak{m}$. Then $\operatorname{dim} E / x E \geq r-1$.

Sketch of a proof. By Nakayama's Lemma $E / x E \neq 0$. So we have nothing to show when $r=1$. So assume $\operatorname{dim} E \geq 2$. Let $P$ be a prime ideal in $A$ with $P \in \operatorname{Supp}(E)$ and $\operatorname{dim} A / P=r$. If $x \in P$ then choose $\mathfrak{q}=P$. Otherwise choose $\mathfrak{q}$ minimal over $(P, x)$. Then by Nakayama's Lemma $(E / x E)_{\mathfrak{q}} \neq 0$. The result follows.

An easy consequence of the above result is:

Corollary 8.5. Let $(A, \mathfrak{m})$ be a Cohen-Macaulay local ring and let $M, N$ be $M C M$ $A$-modules with $\operatorname{dim} \operatorname{Ext}_{A}^{1}(M, N)=r \geq 1$. Let $x \in \mathfrak{m}$ be a non-zero divisor in $A$. Set $B=A /(x), \bar{M}=M / x M$ and $\bar{N}=N / x N$. Then $\operatorname{dim} \operatorname{Ext}_{B}^{1}(\bar{M}, \bar{N}) \geq r-1$.

Sketch of a proof. Note that $x$ is $M \oplus N$-regular. The exact sequence $0 \rightarrow N \stackrel{x}{\rightarrow}$ $N \rightarrow \bar{N} \rightarrow 0$ induces an exact sequence

$$
\operatorname{Ext}_{A}^{1}(M, N) \stackrel{x}{\rightarrow} \operatorname{Ext}_{A}^{1}(M, N) \rightarrow \operatorname{Ext}_{A}^{1}(M, \bar{N})=\operatorname{Ext}_{B}^{1}(\bar{M}, \bar{N})
$$

The result now follows from Lemma 8.4

We now give

Proof of Theorem 8.1. We have already proved the result for $c=1$. Assume $c \geq 2$. Set $R=Q /\left(f_{1}\right)$. Then $M=R /(g)$ and $N=R /\left(g^{i-1} h\right)$ are MCM $A$ modules with Cohen-Macaulay associated graded modules. Note that $f_{2}^{*}, \cdots, f_{c}^{*}$ are $G(R)$-regular and so $G(M), G(N)$ regular sequence. Set $\bar{M}=M /\left(f_{2}, \cdots, f_{c}\right)$ and $\bar{N}=N /\left(f_{2}, \cdots, f_{c}\right)$. Then $\bar{M}$ and $\bar{N}$ are maximal Cohen-Macaulay $A$-modules with Cohen-Macaulay associated graded modules. Using 8.3 and 8.5 we get that $\operatorname{dim} \operatorname{Ext}_{A}^{1}(\bar{M}, \bar{N}) \geq 1$. The result follows from 7.1

\section{Small Dimensions}

In this section we show that if $(A, \mathfrak{m})$ is Cohen-Macaulay of dimension two with $G(A)$ Cohen-Macaulay then there exists a MCM $A$-module $M$ with $G(M)$ CohenMacaulay. If $A$ is not an isolated singularity then we show that there exists an

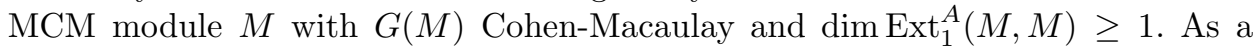
consequence we prove weak Brauer-Thrall II for non-isolated singularities of dimensions 1,2 . We also prove a few preliminary results on Ulrich modules over a one dimensional Cohen-Macaulay local ring.

9.1. The following result showing existence of Ulrich modules in one-dimensional Cohen-Macaulay local rings is well-known. We give a proof due to lack of a reference.

Proposition 9.2. Let $(A, \mathfrak{m})$ be a one-dimensional Cohen-Macaulay local ring and let $E$ be a MCM A-module. Then for all $n \gg 0$ the modules $\mathfrak{m}^{n} E$ are Ulrich A-modules.

Proof. As $\operatorname{dim} E=1$ there exists $n_{0}$ with $e=e(E)=\mu\left(\mathfrak{m}^{n} E\right)$ for all $n \geq n_{0}$. Fix $n \geq n_{0}$. Set $M=\mathfrak{m}^{n} E$. Then $M$ is a MCM $A$-module. Furthermore $e(M)=e$. By construction $\mu(M)=e$. Thus $M$ is an Ulrich $A$-module.

The following result is required in section 10 . 
Proposition 9.3. Let $(A, \mathfrak{m})$ be a one-dimensional Cohen-Macaulay local ring which is not an isolated singularity (equivalently $A$ is not reduced). Then there exists an Ulrich A-module $M$ with $\operatorname{dim} \operatorname{Ext}_{A}^{1}(M, M)=1$

Proof. Let $P$ be a minimal prime of $A$ with $A_{P}$ not a regular local ring (equivalently $A_{P}$ is not a field). Let $M$ be an Ulrich $A / P$-module. Then $M$ is also an Ulrich $A$-module. Notice $M_{P}=\kappa(P)^{r}$ for some $r \geq 1$ (here $\kappa(P)$ is the residue field of $\left.A_{P}\right)$.

We have

$$
\operatorname{Ext}_{A}^{1}(M, M)_{P}=\operatorname{Ext}_{A_{P}}^{1}\left(M_{P}, M_{P}\right)=\operatorname{Ext}_{A_{P}}^{1}\left(\kappa(P)^{r}, \kappa(P)^{r}\right) \neq 0 .
$$

The last assertion holds since $A_{P}$ is not a regular ring. Thus $\operatorname{dim}_{A} \operatorname{Ext}_{A}^{1}(M, M) \neq 0$. The result follows.

We now show one of the main results of this section.

Theorem 9.4. Let $(A, \mathfrak{m})$ be a two dimensional Cohen-Macaulay local ring with $G(A)$ Cohen-Macaulay. Then there exists an MCM A-module $M$ with $G(M)$ CohenMacaulay.

Proof. Let $B$ be a one-dimensional Cohen-Macaulay quotient of $A$ and let $E$ be an Ulrich $B$-module. Let $M=\operatorname{Syz}_{1}^{A}(E)$. We claim that $G(M)$ is Cohen-Macaulay. Without loss of any generality we may assume that the residue field $k$ of $A$ is infinite. Let $x$ be $A \oplus M \oplus E$-superficial. Then $E / x E=k^{r}$ for some $r \geq 1$. Then $M / x M=\mathfrak{n}^{r}$ where $\mathfrak{n}$ is the maximal ideal of $C=A /(x)$. Note that $G(C)$ is CohenMacaulay and so $G(\mathfrak{n})$ is Cohen-Macaulay. Thus $G(M / x M)$ is Cohen-Macaulay. By Sally descent we have $G(M)$ is Cohen-Macaulay.

We will also need the following result.

Proposition 9.5. Let $(A, \mathfrak{m})$ be a two dimensional Cohen-Macaulay local ring with $G(A)$ Cohen-Macaulay. Assume $A$ is not an isolated singularity. Then there exists an $M C M$ A-module $M$ with $G(M)$ Cohen-Macaulay and $\operatorname{dim}_{\operatorname{Ext}_{1}^{A}}^{A}(M, M) \geq 1$.

Proof. Let $P$ be a height one prime with $A_{P}$ not regular. Let $E$ be an Ulrich $A / P$ module, By proof of Theorem 9.4 we get that $M=\operatorname{Syz}_{1}^{A}(E)$ has $G(M)$ CohenMacaulay.

Claim: $\operatorname{dim} \operatorname{Ext}_{A}^{1}(M, M) \geq 1$.

It suffices to prove that $\operatorname{Ext}_{A}^{1}(M, M)_{P} \neq 0$. Notice $E_{P}=\kappa(P)^{r}$ for some $r \geq 1$ (here $\kappa(P)$ is the residue field of $A_{P}$ ). It follows that $M_{P} \cong \mathfrak{n}^{r} \oplus A_{P}^{s}$ where $\mathfrak{n}$ is the maximal ideal of $A_{P}$. By Lemma 9.6 we have that $\operatorname{Ext}_{A_{P}}^{1}(\mathfrak{n}, \mathfrak{n}) \neq 0$. This proves the result.

We need the following result in the proof of Proposition 9.5 I believe that this already known to the experts. We give a proof due to lack of a suitable reference.

Lemma 9.6. Let $(S, \mathfrak{n})$ be a one dimensional non-regular local ring. Then $\operatorname{Ext}_{S}^{1}(\mathfrak{n}, \mathfrak{n}) \neq 0$.

Proof. We first assert that $\operatorname{injdim}_{S} \mathfrak{n}=\infty$. Suppose if possible injdim $\mathfrak{n}<\infty$. Let $x \in \mathfrak{n} \backslash \mathfrak{n}^{2}$ be $S$-regular. So it is also $\mathfrak{n}$-regular. Set $R=S /(x)$ and $k=$ residue field of $R$. As $\operatorname{injdim}_{S} \mathfrak{n}<\infty$ we get $\operatorname{injdim}_{R} \mathfrak{n} / x \mathfrak{n}<\infty$. We have a split exact sequence of $R$-modules:

$$
0 \rightarrow k \rightarrow \mathfrak{n} / x \mathfrak{n} \rightarrow \mathfrak{n} /(x) \rightarrow 0 .
$$


It follows that $\operatorname{injdim}_{R} k<\infty$. So $R$ is regular and as $x \in \mathfrak{n} \backslash \mathfrak{n}^{2}$ we get that $S$ is also regular, a contradiction.

Suppose if possible $\operatorname{Ext}_{S}^{1}(\mathfrak{n}, \mathfrak{n})=0$. By applying the functor $\operatorname{Hom}_{S}(-, \mathfrak{n})$ to the exact sequence $0 \rightarrow \mathfrak{n} \rightarrow S \rightarrow k \rightarrow 0$ we get $\operatorname{Ext}_{S}^{2}(k, \mathfrak{n})=0$. This is a contradiction as $\operatorname{dim} S=1$ and $\operatorname{injdim} \mathfrak{n}=\infty$, see [2] exercise problem 3.5.12(b).

An easy consequence of our previous results is weak Brauer-Thrall II for associated graded modules in dimensions one and two.

Theorem 9.7. Let $(A, \mathfrak{m})$ be a Cohen-Macaulay local ring of dimension one or two. Assume $A$ is not an isolated singularity and that $G(A)$ is Cohen-Macaulay. Then weak Brauer-Thrall II holds for associated graded modules of A.

Proof. This follows from $9.3,9.5$ and 7.1 .

\section{WEAK BRAUER-Thrall II FOR UlRICH MOdules AND FOR RElative COMPLETE INTERSECTIONS}

In this section we discuss our results regarding weak Brauer-Thrall II for Ulrich modules and for relative complete intersections. In dimension one we have the following:

Proposition 10.1. Let $(A, \mathfrak{m})$ be a one-dimensional Cohen-Macaulay local ring with $G(A)$ Cohen-Macaulay. Assume $A$ is not an isolated singularity. Then $A$ satisfies weak Brauer-Thrall II for Ulrich modules.

Proof. This follows from 9.3 and 7.3 .

The main result of this section is

Proposition 10.2. Let $(A, \mathfrak{m})$ be a Cohen-Macaulay local ring with $G(A)$ CohenMacaulay. Assume $A$ has an Ulrich module $U$. Let $r \geq 1$ and let $B$ be either $A\left[X_{1}, \ldots, X_{r}\right]_{\left(\mathfrak{m}, X_{1}, \ldots, X_{r}\right)}$ or $A\left[\left[X_{1}, \ldots, X_{r}\right]\right]$. Then $B$ satisfies weak Brauer-ThrallII for Ulrich modules.

Proof. Note that $B$ is a flat extension of $A$ with an $r$-dimensional fiber. Also note that $M=U \otimes_{A} B$ is an Ulrich $B$-module with $\operatorname{dim}_{\operatorname{Ext}_{B}^{1}}(M, M) \geq r$ (see [2, Theorem A.11(b)]). The result now follows from 7.3 .

Finally we prove weak Brauer-Thrall II for relative complete intersections.

Theorem 10.3. Let $(R, \mathfrak{n})$ be a Cohen-Macaulay local ring having a non-free $M C M$ module $E$ with $G(E)$ Cohen-Macaulay. Also assume $G(R)$ is Cohen-Macaulay. Let $r \geq 1$ and let $B=A\left[\left[X_{1}, \ldots, X_{r}\right]\right]$ or $B=R\left[X_{1}, \ldots, X_{r}\right]_{\left(\mathfrak{n}, X_{1}, \ldots, X_{r}\right)}$. Note $G(B)$ is Cohen-Macaulay. Let $0 \leq l \leq r-1$ and let $g_{1}, \ldots, g_{l}$ be such that $g_{1}^{*}, \ldots, g_{l}^{*}$ is $G(B)$ regular. Set $A=B /\left(g_{1}, \ldots, g_{l}\right)$. Then A satisfies weak Brauer-Thrall II for associated graded modules.

Proof. Set $N=E \otimes_{R} B$. Notice $N$ is a non-free maximal Cohen-Macaulay $B$ module with $G(N)$ Cohen-Macaulay. $B$ is a flat extension of $A$ with an $r$-dimensional fiber. We have $\operatorname{dim} \operatorname{Ext}_{B}^{1}(N, N) \geq r$ (see [2, Theorem A.11(b)]).

As $G(N)$ is a MCM $G(B)$-module we have that $g_{1}^{*}, \ldots, g_{l}^{*}$ is $G(N)$ regular. Set $M=N /\left(g_{1}, \ldots, g_{l}\right) N$. We note that $G(M)$ is Cohen-Macaulay. By 8.5 we get that $\operatorname{dim} \operatorname{Ext}_{A}^{1}(M, M) \geq 1$. The result follows from Theorem 7.1 


\section{REFERENCES}

[1] J. P. Brennan, J. Herzog and B. Ulrich, Maximally generated Cohen-Macaulay modules, Math. Scand. 61 (1987), no. 2, 181-203.

[2] W. Bruns and J. Herzog, Cohen-Macaulay Rings, revised edition, Cambridge Stud. Adv. Math., vol. 39, Cambridge University Press, Cambridge, (1998).

[3] E. Dieterich, Reduction of isolated singularities, Comment. Math. Helv. 62 (1987), 654-676.

[4] David Eisenbud, Homological algebra on a complete intersection, with an application to group representations, Trans. Amer. Math. Soc. 260 (1980), no. 1, 35-64.

[5] J. Herzog, B. Ulrich and J. Backelin, Linear maximal Cohen-Macaulay modules over strict complete intersections, J. Pure Appl. Algebra 71 (1991), no. 2-3, 187-202.

[6] C. Huneke and G. J. Leuschke, Two theorems about maximal Cohen-Macaulay modules, Math. Ann. 324 (2002), no. 2, 3914-04.

[7] S. B. Iyengar; G. J. Leuschke; A. Leykin; C. Miller; E. Miller; A. K. Singh and U. Walther, Twenty-four hours of local cohomology. Graduate Studies in Mathematics, 87. American Mathematical Society, Providence, RI, 2007.

[8] H. Matsumura, Commutative ring theory, second ed., Cambridge Studies in Advanced Mathematics, vol. 8, Cambridge University Press, Cambridge, 1989.

[9] T. J. Puthenpurakal, Hilbert-coefficients of a Cohen-Macaulay module, J. Algebra 264 (2003), no. $1,82-97$.

[10] - The Hilbert function of a maximal Cohen-Macaulay module, Math. Z. 251 (2005), no. 3, 551-573.

[11] - Ratliff-Rush filtration, regularity and depth of higher associated graded modules. I, J. Pure Appl. Algebra 208 (2007), no. 1, 159-176.

[12] _ A function on the set of isomorphism classes in the stable category of maximal Cohen-Macaulay modules over a Gorenstein ring: with applications to liaison theory, Math. Scand. 120 (2017), no. 2, 161-180

[13] Growth of Hilbert coefficients of syzygy modules, J. Algebra 482 (2017), 131158.

[14] Symmetries and Connected components of the AR-quiver of a Gorenstein local ring. Algebras and Representation theory (to appear).

[15] J. J. Rotman, An introduction to homological algebra, Pure and Applied Mathematics, 85. Academic Press, Inc. New York-London, 1979.

[16] Y. Yoshino, Brauer-Thrall type theorem for maximal Cohen-Macaulay modules, J. Math. Soc. Japan 39 (1987), no. 4, 719-739

[17] _ Cohen-Macaulay modules over Cohen-Macaulay rings, London Mathematical Society Lecture Note Series, 146. Cambridge University Press, Cambridge, 1990.

Department of Mathematics, Indian Institute of Technology Bombay, Powai, Mumbai 400 076, INDIA

E-mail address: tputhen@math.iitb.ac.in 